\title{
Pandemic influenza outbreak detection and response in Farta district, South Gondar zone, Amhara region, Ethiopia, 2016
}

\author{
Alie Ayal Ejigu' ${ }^{1 *}$, Mekonnen Assefa ${ }^{2}$, Marelign Tilahun ${ }^{2}$, Feleke Tilahun Zewdu ${ }^{3}$ \\ ${ }^{1}$ Department of Public Health, Amhara Public Health Institution, Amhara, Ethiopia, ${ }^{2}$ Department of Public Health, Mph/Epidemiology and \\ Biostatistics, College of Medicine and Health Science, Debretabor University, Amhara, Ethiopia, ${ }^{3}$ Department of Tropical Dermatology, \\ Boru Meda Hospital, South Wollo Zone, Amhara, Ethiopia
}

\begin{abstract}
Background: Influenza is the most contagious respiratory diseases having significant public health problem. It is associated with higher morbidity and mortality during outbreak, especially among elderly and children. The aim of the study was to investigate the outbreak, magnitude, identify risk factors, and implement public health control measures.

Methods: We conduct case-control study in Farta district from February 12 to $29^{\text {th }}$ February 2016 . We selected 172 cases and 172 controls (1:1). We used face-to-face interview using structured questionnaire. Data were entered and analyzed using Epi-info7 and SPSS. We used logistic regression analysis to explore predictors of influenza.

Results: From February 12 to February 30, 2016, we identified 172 cases (attack rate, 10.38/10oo) and two deaths. From total cases, $11(6.4 \%)$ confirmed and $23(13.4 \%)$ admitted cases were reported. Female cases were $51.74 \%$. Majority affected age groups were $<5$ and 15-44 years with attack rates of 16.81 and 10.36 per 1000 risk populations, respectively. In multivariable analysis (adjusted odds ratio [95\% confidence intervals]), being female $(2.76[1.11,8.32])$, family size $\geq 5(3.26[1.12,14.59])$, presence of traveling history to epidemic area before 7 days before onset of the illness (10.57 [1.34, 42.79]), and having knowledge to influenza illness (o.13 [0.02, 0.91]) were associated factors to the respective variables.

Conclusion: Most of the cases were female and 15-44 year's age. Being female, living in large family size and presence of traveling history to epidemic area before 7 days before onset of the illness were statistically significant risk factors; whereas, having knowledge to influenza illness was protective factors. Health education should be continued to successfully contain the outbreak.

Key words: Ethiopia, Farta district, influenza, outbreak, risk factor
\end{abstract}

\section{INTRODUCTION}

Influenza is a contagious respiratory illness caused by the influenza virus. There are three types of influenza viruses $A, B$, and C. Influenza is divided into subtypes based on two proteins on the surface of the virus. Only some influenza, a subtype (i.e., influenza A [H1N1] and $\mathrm{H}_{3} \mathrm{~N}_{2}$ ) is currently, in general, circulation among people. ${ }^{[1]}$

Influenza is manifested by fever, cough sore throat, runny nose, muscle/body ache, headache, fatigue/tiredness, joint pain, and sometimes vomiting and diarrhea. ${ }^{[2]}$

Any person with acute respiratory infection with fever $\geq 38^{\circ} \mathrm{C}$ and cough or sore throat and onset within the past 10 days is the standard case definition of influenza likeliness. ${ }^{[3]}$

Influenza viruses are spread from person to person by respiratory droplets generated when an infected person coughs, sneezes, or talks in close proximity to an uninfected person and by touching contaminated objects (e.g., a doorknob). Most healthy adults who are ill with influenza may be able to infect other people beginning 1 day before symptoms develop and up to 5-7 days after becoming sick. Influenza usually comes on suddenly, 1-4 days after the virus enters the body. ${ }^{[1]}$

The WHO estimates that annual influenza epidemics affect 5-15\% of the global population, resulting in severe illness in 3-5 million patients and causing 250,000-500,000 deaths worldwide. In addition to these annual epidemics, the influenza a virus has caused three major global pandemics during the $20^{\text {th }}$ century: The Spanish flu in 1918, Asian flu in 1957, and Hong Kong flu in 1968-1969. ${ }^{[4]}$

It is an estimated 28,000-111,500 deaths annually among children aged $<5$ years, with $99 \%$ of these deaths occurring in developing countries. In Africa, respiratory infections rank among the leading causes of morbidity and mortality. ${ }^{[5]}$

Even in a non-pandemic year, influenza is estimated to cause between 250,000 and 500,000 deaths worldwide. ${ }^{[6]}$

Address for correspondence:

Alie Ayal Ejigu, Department of Public Health, Amhara Public Health Institution, Amhara, Ethiopia. E-mail: semreabe21@gmail.com

Received: 05-04-2018 Revised: 10-05-2018 Accepted: 20-06-2018 
The overall test positivity rate of the influenza disease was $21.7 \%$ in 15 countries of Africa ${ }^{[7]}$ and $6.8 \%$ in Ethiopia. ${ }^{[5]}$

Females accounted more than half of the patients with respiratory illness (54.3\%), H1N1 virus (59.3\%), and the mean annual rate of influenza-associated inpatient mortality was 1.5 deaths per 100,000 population. There were 7.6 influenza-associated death per 100,000 children aged $<5$ years, but only 0.2 per 100,000 individuals aged $\geq 5$ years. ${ }^{[8]}$

Influenza-like illness (ILI) is one of the priority diseases that are immediately reportable in public health emergency managements (PHEM) system in Ethiopia. On February 12, 2016, Zonal Health Department PHEM unit received ILI report from Farta district through the routine immediately surveillance report. From February 12 to 30, 2016, Zonal Health Department sends the team that includes epidemiologists from Debretabor University to investigate and responds to the problem. The investigation team conducted a case-control study in Farta district, South Gondar Zone. The aim of the study was to verify existence, to describe magnitude, and to identify etiological agents and risk factors associated with pandemic influenza outbreak and undertake appropriate public health control measures.

\section{MATERIALS AND METHODS}

\section{Study Area}

The investigation was conducted in Farta district. The district is located $100 \mathrm{KM}$ from Bahir Dar and $666 \mathrm{KM}$ from the capital city of Addis Ababa in the North-West part of Ethiopia.

The district is found in South Gondar Zone. Administratively, the district is divided into 43 kebeles with an estimated population, size of 276,$144 ; 50.5 \%$ were females based on 2007 census projection. There are a total of 112 health extension workers in the district, within a total of 54 health posts, 10 health center, and 4 private clinics.

\section{Study Period}

The study was conducted from February 12 to 30, 2016.

\section{Study Design}

Unmatched case-control study design with the ratio of 1:1 was used.

\section{Target Population}

All populations in the outbreak affected areas of the district, where cases and controls found.

\section{Study Population}

The study population were any resident of Farta district whom the WHO case definition of ILI during the study period consider as a case and any resident of Farta district during the study period who did not develop signs and symptoms of ILI during the study period considered as a control.

\section{Sample Size Determination}

All case-patients enrolled in the study due to small number of cases. We used significant increase in terms of precision or statistical power assumption. Thus, we used 1:1 case-control ratios and calculated a total sample size was 172 cases and 172 controls (344 study subjects).

\section{Sampling Procedure}

We selected all cases in the affected district using line list. For controls, households were selected using simple random sampling method at each affected kebele on the sampling frame of community health information system family folder at health post-level. If more than one eligible lived in the selected household, one control was selected by the lottery method.

\section{Data Collection}

We collected data using a structured questionnaire adapted from influenza case-based format to interview both cases and controls; the data collected include identification, demographic, risk factors, and clinical information.

\section{Case Finding and Identification}

The team carried out house-to-house active search of cases using the centers for disease control (CDC)/standard case definition for pandemic influenza.

\section{Data Analysis}

The collected data were checked and entered on a computer and analyzed using EPI 7. Bivariate and multivariate logistic regression analysis was conducted using SPSS to find association of influenza illness with risk factors and outcome was measured and tested using OR, 95\% confidence interval (CI), and $P$-value.

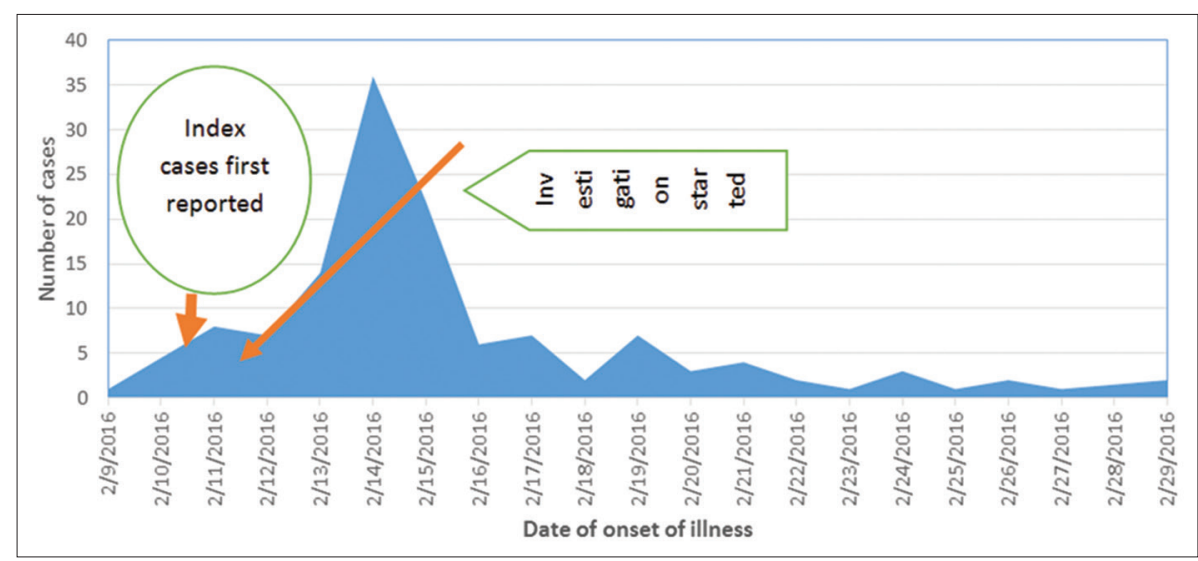

Figure 1: Influenza illness outbreak EPI curve by date of onset in Farta District, 2016 


\section{Ethical Issue}

Outbreak investigation is one part of public health response, thus no need of institutional review board approval. However, permission letter was obtained from South Gondar Zonal Health Department and Farta district health office. We introduced ourselves and explained the purpose of the study using specific statements in a standard procedure. Verbal consent was obtained from participants or from their parents to participate in the study. Confidentiality was assured and no personal details were recorded or produced on this documentation.

\section{Enrollment of Cases and Controls}

A. Cases: Those that have clinical signs/symptoms of pandemic influenza based on CDC case definitions that were either laboratory confirmed or epidemiologically linked to the laboratory confirmed cases.

B. Controls: Those neighbors and siblings who were not show clinical manifestation of pandemic influenza during the study period were taken as controls irrespective of their demographic characteristics.

\section{Operational Definition Case definition}

An ILI, any individual with fever, cough, sore throat, shortness of breath, difficulty of breathing, and chest pain and/or has history of contact during the 7 days before the onset of symptoms with sick or dead birds, including chickens. ${ }^{[9]}$

\section{Case classification}

Possible case is any person meeting the clinical criteria ILI. Probable case is any person meeting the clinical criteria ILI and an epidemiological link. Confirmed case is any person meeting the clinical ILI and positive results in a national influenza laboratory.

\section{Suspected case}

A person with acute febrile respiratory illness: Fever, cough, sore throat, shortness of breath, difficulty in breathing, or chest pains with onset: Within 7 days of close contact with a person who is a probable or confirmed case of the new H1N1 virus infection, or within 7 days of travel to a community internationally where there have been one or more confirmed novel H1N1 cases or resides in a community where there are confirmed new influenza cases.

\section{Probable case}

An individual with an influenza test that is positive for H1N1 but is unsubtypable by reagents used to detect seasonal influenza virus infection.

\section{OR}

An individual with a clinically compatible illness or who died of an unexplained acute respiratory illness is considered to be epidemiologically linked to a probable or confirmed case.

\section{Confirmed case}

An individual with laboratory confirmed new H1N1 virus infection by real-time polymerase chain reaction (RT-PCR).

\section{Environmental investigation}

We tried to ask the community presence of any animal death, social ceremony in the area which creates favorable condition for spread of the disease.

\section{Variables \\ Dependent variable}

Influenza illness.

$$
\begin{aligned}
& \text { Independent variables } \\
& \text { - Age } \\
& \text { - Sex } \\
& \text { - Knowledge of influenza } \\
& \text { - Family size } \\
& \text { - Educational status } \\
& \text { - Contact history } \\
& \text { - Marital status } \\
& \text { - Occupational status } \\
& \text { - Travel history. }
\end{aligned}
$$

\section{RESULTS}

\section{Descriptive Epidemiology}

From February 12 to 29, 2016, we identified a total of 172 cases (11 confirmed and 161 epidemiologically linked). Overall, attack rate was 10.38 per 1000 risk populations [Figure 1].

\section{Laboratory Investigation}

From 27 ill patients, throat swab specimens were collected. Specimens were stored and shipped to national influenza laboratory in viral transport media at $2-8 \mathrm{C}^{\circ}$, and tested by RTPCR for influenza viruses. 11 of 27 (40.74\%) were positive for "Pdm09/H1 H1N1" subtype.

\section{Case Distribution by Place}

Among 43 kebeles, only three were affected. Attack rates per 1000 risk populations were16.59 in Megindi, 11.18 in D/Eyesus, and 2.31in M/Mariyam.

\section{Case Distribution by Person}

Cases and controls median age was 21 years (range 7 months-70 years) and 30 years (range 1 year-75 years), respectively. Female cases were 89 (51.74\%). <5 years were considerably more affected than the others (attack rate was 16.81 per 1000 risk populations) and followed by aged 15-64 years were 10.36 per 1000 risk populations [Table 1].

\section{Cases Distribution by Time}

We observed a triple peak in different times during the outbreak; this shows propagated source of transmission. The duration of outbreak is starting from February 12 to 30.

\section{Clinical Characteristics of Influenza Illness}

The major manifestation of the signs and symptoms during the outbreak was characterized by high-grade fever (100\%), cough $(85.6 \%)$, headache $(57.45 \%)$, sore throat (18\%), and myalgia (11.2\%).

\section{Analytic Epidemiology}

In multivariable analysis (adjusted odds ratio [AOR] [95\% CI]), being female $(3.76[1.23,11.52])$, family size $\geq 5$ (4.56 [1.24, 16.79]), having contact history with ILI (5.57 [1.64, 18.89]), living unventilated house $(0.14$ [0.04, 0.42]), and having knowledge of ILI $(0.1[0.01,0.91])$ were statistically significant factors [Table 2]. 


\begin{tabular}{|c|c|c|c|c|c|c|}
\hline $\begin{array}{l}\text { Age } \\
\text { group }\end{array}$ & $\begin{array}{c}\text { Population at } \\
\text { risk }\end{array}$ & $\begin{array}{c}\text { Case } \\
\text { frequency }\end{array}$ & $\begin{array}{l}\text { Cumulative } \\
\text { frequency }\end{array}$ & $\begin{array}{c}\text { Relative } \\
\text { frequency }(\%)\end{array}$ & $\begin{array}{c}\text { Cumulative relative } \\
\text { frequency (\%) }\end{array}$ & $\begin{array}{c}\text { Age-specific attack } \\
\text { rate } / 1000\end{array}$ \\
\hline$<5$ & 2.320 & 39 & 39 & 22.67 & 22.67 & 16.81 \\
\hline $5-14$ & 4.971 & 20 & 59 & 11.63 & $34 \cdot 30$ & 4.02 \\
\hline $15-64$ & 8.782 & 91 & 150 & 52.90 & 87.20 & 10.36 \\
\hline$\geq 65$ & 497 & 22 & 172 & 12.79 & 100.00 & 4.42 \\
\hline Total & 16.570 & 172 & - & 100.00 & - & 10.38 \\
\hline
\end{tabular}

ILI: Influenza-like illness

\begin{tabular}{|c|c|c|c|c|c|}
\hline Variable & Case $n=172$ & Control $n=172$ & COR $(95 \%$ CI $)$ & AOR (95\% CI) & $P$-Value \\
\hline \multicolumn{6}{|l|}{ Sex } \\
\hline Female & $89(51.74)$ & $71(41.28)$ & $2.26(1.24,7.36)$ & $2.76(1.11,8.32)$ & 0.02 \\
\hline Male & $83(48.26)$ & $101(58.72)$ & + & + & + \\
\hline \multicolumn{6}{|l|}{ Family size } \\
\hline$\leq 2$ & $37(21.43)$ & $72(41.96)$ & + & + & + \\
\hline $3-4$ & $31(17.86)$ & $54(31.25)$ & $1.12(0.22,3.27)$ & $0.79(0.2,3.89)$ & 0.77 \\
\hline$\geq 5$ & $104(60.71)$ & $46(26.79)$ & $3.12(1.37,11.53)$ & $3.26(1.12,14.59)$ & 0.02 \\
\hline \multicolumn{6}{|c|}{ Traveling history to epidemic area } \\
\hline Yes & $103(59.88)$ & $9(5.23)$ & $2.84(1.71,3.67)$ & $5.47(1.34,16.49)$ & 0.006 \\
\hline No & $69(41.12)$ & $163(94.77)$ & + & + & + \\
\hline \multicolumn{6}{|c|}{ Presence of cases in family/community } \\
\hline Yes & $148(86.05)$ & $46(26.74)$ & $5.99(1.64,9.67)$ & $10.57(1.54,21.79)$ & $<0.001$ \\
\hline No & $24(13.95)$ & $126(73.26)$ & + & + & + \\
\hline \multicolumn{6}{|c|}{ Having knowledge to influenza } \\
\hline Yes & $48(27.91)$ & $122(70.93)$ & $0.06(0.01,0.57)$ & $0.13(0.01,0.91)$ & $<0.001$ \\
\hline No & $124(72.09)$ & $50(29.07)$ & + & + & + \\
\hline
\end{tabular}

+: Reference, Cl: Confidence intervals, AOR: Adjusted odds ratio, COR: Crude odds ratio

\section{Action Taken}

We conducted brief discussion with district administrative and health official, and then the team engaged in activating the district epidemic response task force to participate in active case detection and educating the community to control the epidemic. Throat swabs were taken from patients for confirmation of the outbreak; isolation of admitted cases in health facilities to prevent crosstransmission; mobilizing clinical management, investigation, and social mobilization teams; technical guidance to strengthen the coordination mechanism to stakeholders (to involve sectors such as education and communication); epidemiologic investigation conducted including a case-control study; distribution of infection prevention materials to patient and care providers (masks); and implementation of strike handwashing procedures, and media briefing in different areas to create awareness about prevention mechanisms, strength, surveillance, and supportive care at home.

\section{DISCUSSION}

Everyone can get the flu, but certain age groups had increased risk of the disease and serious complications. This includes children younger than 5, pregnant women, and people who have medical conditions. $^{[10]}$

Investigation report identified a total of 172 ILI cases with attack rate of 10.38 per 1000 risk populations. Most of the cases were female $(51.74 \%)$ and under-five children with age-specific attack rate of 16.81 per 1000 risk population which was slightly higher than the overall attack rate. The main manifestation of the signs and symptoms during the outbreak was also characterized by high-grade fever, cough, headache, and sore throat. This is in line with different literatures. ${ }^{[9]}$ Laboratory for etiologic causes confirmation for influenza; 11 (40.74\%) suspected ILI were positive for influenza $\mathrm{A}$, which were higher positivity rates compared with the study conducted in routine influenza sentinel surveillance in Ethiopia, 2008-2010. ${ }^{[5]}$

Our study found that attack rate was to some extent higher in study subjects under 5 years old. Being female was significantly associated with ILI; the odd of developing ILI was 2.76 times among females compared to males. The possible reason might be females were adjusted for living with children or for daily contact with groups of children or caregivers to patients at home. Having family size equal or $>5$ in household was significantly associated with ILI; the odd of developing disease was 3.26 times higher compared with having family size $\leq 2$. The possible reason might be over condensed family size or confined space increased person density in the household and suitable to transmission of infection during sneezing, cough, or talk; consistent with the study done in Victoria Australia. ${ }^{[11]}$ People who had the presence of cases in family/community with suspected influenza illness were 10.57 times more likely to develop influenza illness than people had not the presence of cases in family/community. The possible reason could, in fact, cross-contamination play a great role in infectious diseases or direct contact may have increased impact on transmission through aerosols or by indirect contact with contaminated with virus-containing fomites on environmental surfaces consistent with other studies. ${ }^{[11]}$ Having knowledge of about influenza illness transmission and prevention significantly 
reduces the risk of acquiring influenza illness by almost $87 \% \mid 1$ AOR|* 100 compared with others.

\section{Limitation of the Study}

The study design was prone to selection, specifically in the control group. The finding may not be representative for the entire population.

\section{CONCLUSION AND RECOMMENDATION}

Most of the cases were female and under-five children. 11 of 27 $(40.74 \%)$ were confirmed by PCR test positive for "Pdm09/H1 H1N1" subtype. Being female, living in large family size, traveling history to epidemic area, and having contact history with ILI were statistically significant risk factors; whereas, having knowledge to ILI was protective factors. Hence, health workers should continue health education how to prevent and minimize the spread of the disease using different media to the people regularly.

\section{REFERENCES}

1. Texas Department of State Health Services. FAOs Data Reporting Investigation Immunization Resources Monitoring, Infectious Disease Control Influenza Unit; 2016. p. 1-5.

2. Key Facts about Influenza (Flu) and Flu Vaccine. Available from: http://www.cdc.gov/flu/keyfacts.htm. [Last accessed on 2016 May 06].

3. Kasper MR, Wierzba TF, Sovann L, Putnam SD. Evaluation of an influenza like illness case definition in the diagnosis of influenza among patients with acute febrile illness in Cambodia. BMC Infect Dis 2010;10:320.
4. Federal Democratic Republic of Ethiopia Ministry of Health Pandemic Influenza Preparedness and Response Plan; 2010.

5. Ayele W, Demissie G, Kassa W, Zemelak E, Afework A, Amare $\mathrm{B}$, et al. Challenges of establishing routine influenza sentinel surveillance in Ethiopia, 2008-2010. J Infect Dis 2012;206:S41-5.

6. Adler AJ, Eames KT, Funk S, Edmunds WJ. Incidence and risk factors for influenza-like-illness in the UK : Online surveillance using flusurvey. BMC Infect Dis 2014;14:232.

7. Radin JM, Katz MA, Tempia S, Nzussouo NT, Davis R, Duque $\mathrm{J}$, et al. Influenza surveillance in 15 countries in Africa, 2006-2010. J Infect Dis 2012;206:S14-21.

8. Feikin DR, Ope MO, Aura B, Fuller JA, Gikunju S, Vulule J, et al. The population-based burden of influenza-associated hospitalization in rural western Kenya, 2007-2009. Bull World Health Organ 2012;90:256-63.

9. CDC Novel H1N1/2009 H1N1: Overview of a Pandemic Influenza. Available from: http://www.cdc.gov/h1n1flu/ yearinreview/yir1.htm. [Last accessed on 2016 Feb 02].

10. Hirota Y, Takeshita S, Idek S, Ohkubc A, Fukuyoshl S, Takahash $\mathrm{K}$, et al. Various factors associated with the manifestation of influenza-like illness. Int $\mathrm{J}$ Epidemiol 1992;21:574-82.

11. Belser JA, Maines TR, Tumpey TM, Katz JM, Belser JA, Maines TR, et al. Influenza A virus transmission : Contributing factors and clinical implications. Expert Rev Mol Med 2010;12:e39.

How to cite this Article: Ejigu AA, Assefa M, Tilahun M, Zewdu FT. Pandemic influenza outbreak detection and response in Farta district, South Gondar zone, Amhara region, Ethiopia, 2016. Int. Res. Med. Health Sci., 2018; 1(1):16-20.

Source of Support: Nil, Conflict of Interest: None declared. 